\title{
Hydrostatic Pressure and Electric-field Effects on the Shallow Donor Impurity States in GaAs-Ga ${ }_{0.7} \mathbf{A l}_{0.3} A s$ Quantum-well Wires
}

\author{
J. W. González ${ }^{1}$, N. Porras-Montenegro ${ }^{2}$, and C. A. Duque ${ }^{1}$ \\ ${ }^{1}$ Instituto de Física, Universidad de Antioquia, AA 1226, Medellín - Colombia \\ ${ }^{2}$ Departamento de Física, Universidad del Valle, AA 25360, Cali - Colombia
}

Received on 8 December, 2005

\begin{abstract}
Using a variational procedure within the effective-mass approximation, we have made a theoretical study of the effects of hydrostatic pressure and applied electric fields on the binding energy of a shallow-donor impurity in square-transversal section $\mathrm{GaAs}-\mathrm{Ga}_{0.7} \mathrm{Al}_{0.3}$ As quantum-well wires. The electric field is applied in a plane of the transversal section of the wire and many angular directions are considered. The hydrostatic pressure has been considered both in the direct and indirect gap regime for the $G a_{0.7} A l_{0.3} A s$ material. For the potential barrier that defines the wire region, we consider an $x$-dependent finite and $y$-dependent infinite model. The results we present are for the impurity binding energy and considering different values of the wire dimensions, hydrostatic pressure, applied electric field, and the impurity position in the transversal section of the wire.
\end{abstract}

Keywords: Quantum well wire; Hydrostatic pressure; Electric field

\section{INTRODUCTION}

With the development of experimental techniques and analytical methods, there has been a considerable amount of work devoted to the study of the states of hydrogenic impurities in low-dimensional semiconductor heterostructures such as quantum wells (QWs), quantum-well wires (QWWs) and quantum dots (QDs)[1-6]. The effects of external perturbation such as magnetic fields, hydrostatic pressure or electric fields on the physical properties of low-dimensional systems constitute a subject of considerable interest from both theoretical and technological points of view, due to the importance of these systems in the development of new semiconductor devices and applications.

Some studies have been reported on the applied electricfield dependence of the shallow donor- and/or acceptorimpurity binding energies of the ground and first few excited states in QW and QWW heterostructures with a fixed direction of the external field [3-5]. They have reported the shallow donor and/or acceptor density of impurity states, donorrelated optical absorption and acceptor-related photoluminescence spectra.

The aim of the present work is to study the hydrostatic pressure and Stark effects on confined donor impurities in square-shaped transversal-section $\mathrm{GaAs}-\mathrm{Ga}_{0.7} \mathrm{Al}_{0.3} \mathrm{As}$ QWWs including the angular dependence of the field along the transversal section of the wire. Calculations are performed using a variational procedure within the effective-mass approximation. Image effects are not considered and the dielectric constant and effective masses are taken as the GaAs values along all regions of the heterostructure.

\section{THEORETICAL FRAMEWORK}

Using the effective-mass approximation, the Hamiltonian for a donor impurity confined in a square-transversal-section GaAs- $\mathrm{Ga}_{0.7} \mathrm{Al}_{0.3} \mathrm{As} \mathrm{QWW}$ under hydrostatic pressure $(P)$, and transversal applied electric field $(\vec{F})$ is given by

$$
\begin{array}{r}
H=-\frac{\hbar^{2}}{2 m^{*}(P)} \nabla^{2}+e|\vec{F}|[x \cos (\theta)+y \sin (\theta)] \\
+V(P, x, y)-\frac{e^{2}}{\varepsilon(P) r},
\end{array}
$$

where $r$ is the carrier-impurity distance and $\varepsilon(P)$ is the static dielectric constant [5]. $m^{*}(P)$ is electron effective-mass [5], $\theta$ is the electric field relative angle to the $x$-axis and $V(P, x, y)$ is the potential barrier that confines the carrier in the wire region considered as zero within the wire region, $V_{0}(P)$ for $|x| \geq L_{x}(P) / 2$ [6], and infinite for $|y| \geq L_{y}(P) / 2$, where $L_{x}(P)$ and $L_{y}(P)$ are the pressure dependent transversal dimensions of the wire $[5,7]$.

For the donor wave function we use a variational procedure and assume a hydrogenic-type trial wave function as [3]

$$
\Psi(r)=N \Phi(x, y) e^{-\lambda r}
$$

where $N$ is a normalization constant, $\lambda$ is a variational parameter, and $\Phi(x, y)=f(x) g(y)$ is the eigenfunction of the Hamiltonian in Eq. (1) without the impurity potential, where $[3,5]$

$$
f(x)= \begin{cases}e^{k_{1} x}, & x \leq L_{x}(P) / 2 \\ \alpha A_{i}(x)+\beta B_{i}(x), & |x| \leq L_{x}(P) / 2 \\ e^{-k_{2} x}, & x \geq L_{x}(P) / 2\end{cases}
$$

and

$$
g(y)= \begin{cases}\delta A_{i}(y)+\sigma B_{i}(y), & |y| \leq L_{y}(P) / 2, \\ 0, & y \geq L_{y}(P) / 2 .\end{cases}
$$

Here $A_{i}$ and $B_{i}$ are the usual Airy functions. The donorimpurity binding energy is calculated from the definition

$$
E_{b}=E_{0}-E_{\min }(\lambda),
$$

where $E_{\min }$ is the eigenvalue for the Hamiltonian in (1), minimized with respect to the variational parameter, and $E_{0}$ is the eigenvalue of the same Hamiltonian but without considering the impurity potential term. 


\section{RESULTS AND DISCUSSION}
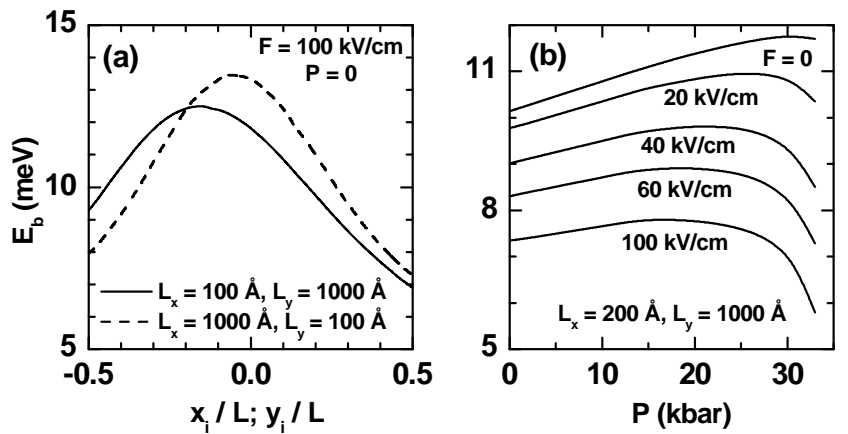

FIG. 1: Binding energy of a donor-impurity in GaAs QWs obtained by consider limiting cases of the transversal-section of the QWWs. In (a) the results are as a function of the impurity position along the growth direction of the QW heterostructure, whereas in (b) the results are for on-center impurity and as a function of hydrostatic pressure. In all cases, the electric field is applied in the growth direction of the QW.

In Fig. 1, we present our results for the binding energy of a donor-impurity in rectangular transversal section QWWs. If $L_{x}$ is large enough (1000 $\AA$ in this case) keeping $L_{y}=100 \AA$ as a constant value, we reproduce the results for the vacuumGaAs-vacuum QW [3], and if $L_{y}$ is large enough (1000 $\AA$ in this case) keeping $L_{x}=100 \AA$ or $200 \AA$ as a constant value, we reproduce the results for the GaAs- $\mathrm{Ga}_{0.7} \mathrm{Al}_{0.3} \mathrm{As} \mathrm{QW}[3$, 5]. In what follows, we will limit our discussion to squaretransversal section QWWs, i.e, to the case in which $L_{x}(P)=$ $L_{y}(P)=L(P)$.

In Fig. 2, we present our results for the binding energy, for an on-axis shallow-donor impurity in a squared-transversal section GaAs- $\mathrm{Ga}_{0.7} \mathrm{Al}_{0.3} \mathrm{As} \mathrm{QWW}$, as a function of the side $(L(P))$ of the wire. As a general feature, we observe that the binding energy increases as the transversal-cross section of the wire decreases. As an effect of the applied electric field, the electron wave function shifts towards the opposite direction of the field, giving origin to a polarization of the system and, consequently, an increase in the expectation value of the electronimpurity distance. Therefore, the impurity binding energy decreases as the Coulomb interaction gets weaker. As one applies hydrostatic pressure, there are direct modifications of three critical parameters, i.e., the dielectric constant, the electron effective-mass and the wire section. Associated with the decrease of the dielectric constant with pressure, an increase in the binding energy is observed. Additionally, due to the difference of pressure coefficients between the well and barrier materials, we observe a slight increase in the binding energy. When the dimensions of the wire are of the order of the GaAs effective Bohr radius, we observe the largest electric-field dependence for $\theta=\pi / 4$ [Fig. 2(b)] and $\theta=0$ [Fig. 2(c)]. Also, the smallest electric-field dependence occurs when the electric field is applied along the direction of the infinite-potential barriers $(\theta=\pi / 2)$ [Fig. 2(a)], in which case the rigid barriers repel the electron wave function towards inside the wire re-
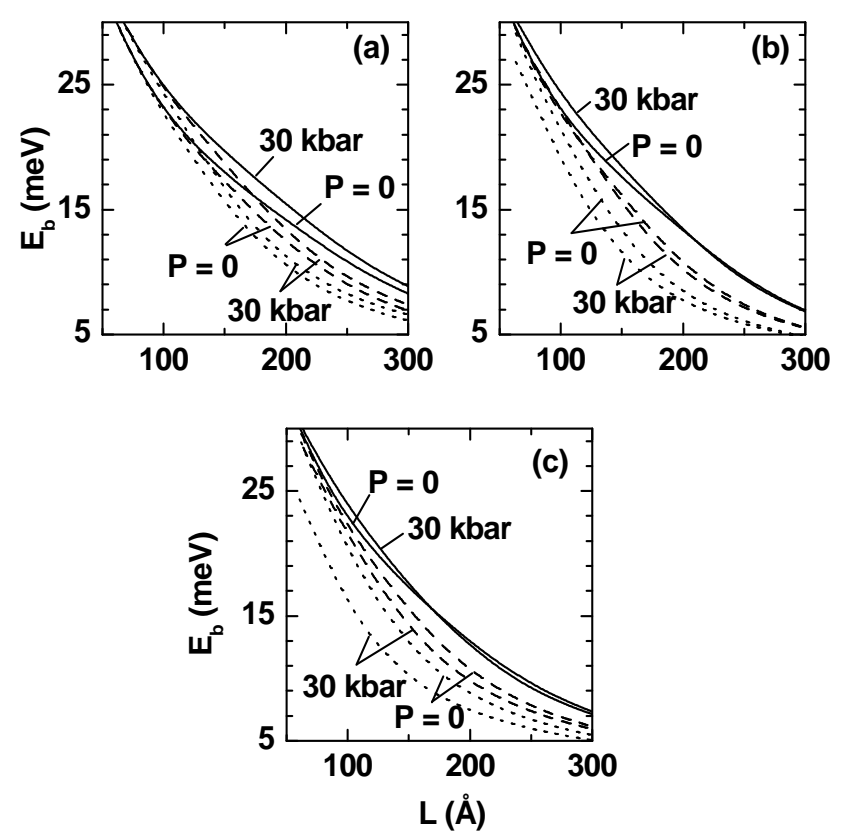

FIG. 2: Binding energy as a function of the side in squaredtransversal section $\mathrm{GaAs}-\mathrm{Ga}_{0.7} \mathrm{Al}_{0.3} \mathrm{As} \mathrm{QWW}$, for two different values of the hydrostatic pressure. Results are for three directions of the applied electric field: $\theta=\pi / 2$ (a), $\theta=\pi / 4$ (b) and $\theta=0$ (c) and for three different values of the applied electric field: $50 \mathrm{kV} / \mathrm{cm}$ (solid lines), $100 \mathrm{kV} / \mathrm{cm}$ (dashed lines), and $200 \mathrm{kV} / \mathrm{cm}$ (dotted lines).

gion. For a low-value of the width of the transversal section $\left(L<a^{*}\right)$, we observe that the structure is in the high-confining geometrical regime and a small dependence with the electric field is observed.

Figure 3 displays the calculated results for the binding energy as a function of the $\left(x_{i}, y_{i}=0\right)$ transversal impurity position in GaAs- $\mathrm{Ga}_{0.7} \mathrm{Al}_{0.3}$ As QWWs. We find that for increasing values of the applied electric field the binding energy shows an increasing asymmetrical behavior with respect to the impurity position, i.e., the symmetry breaks down as the electron wave function is driven in the direction opposite to the applied electric field. One should note that, for a small and fixed value of the electric field [as in Fig. 3(a)], the hydrostatic pressure essentially increases the impurity binding energy by a few meV, without affecting the asymmetry. This very simple behavior can not be observed in the case of high electric fields, as in Fig. 3(b), since as effect of the hydrostatic pressure an increasing or a decreasing behavior on the impurity binding energy can be observed, clearly associated with the impurity position in the transversal section of the wire and with the direction of the applied electric field. The small shift in the binding energy observed for the solid lines in Fig. 3(b) show that, inclusive in the regime of high electric fields and hydrostatic pressures, the infinite potential barrier continues being the dominant factor on the impurity binding energy. Additionally, for effects of clarity we want to stress that in spite of the presence of the applied electric field the solid lines, both in Figs 3(a) and 3(b), are symmetrical with respect to the cen- 
ter of the section of the wire because the impurity is displaced perpendicularly to the direction of the applied electric field.

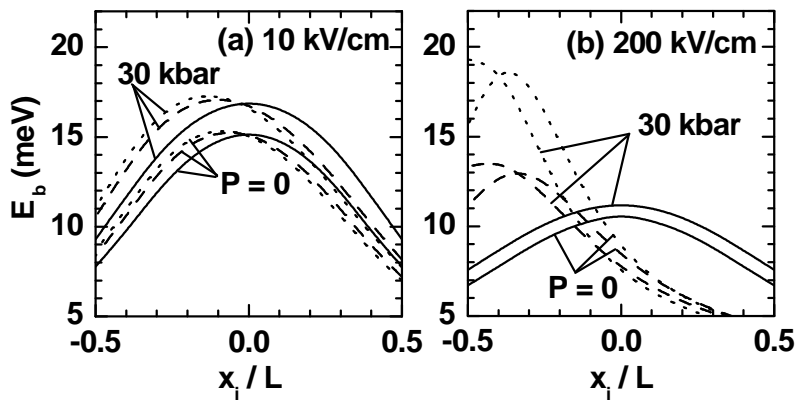

FIG. 3: Binding energy as function of the $\left(x_{i}, y_{i}=0\right)$ transversal impurity position in $\mathrm{GaAs}_{-} \mathrm{Ga}_{0.7} \mathrm{Al}_{0.3} \mathrm{As} \mathrm{QWWs}$. Results are for $L=200 \AA$ and for two different values of the hydrostatic pressure. Electric field values are $10 \mathrm{kV} / \mathrm{cm}$ (a), and $200 \mathrm{kV} / \mathrm{cm}$ (b) and the electric field direction is given by $\theta=\pi / 2$ (solid lines), $\theta=\pi / 4$ (dashed lines), and $\theta=0$ (dotted lines).

In Fig. 4 we present the binding energy as a function of the $\left(x_{i}=0, y_{i}\right)$ shallow-donor impurity positions in a GaAs$\mathrm{Ga}_{0.7} \mathrm{Al}_{0.3} \mathrm{As} \mathrm{QWW}$. We find, as in Fig. 3, the same asymmetries in the binding-energy curves for small values of the applied electric field [Fig. 4(a)]. However, due to the infinitepotential barriers, significative changes in the binding energies are only observed when the impurity is near the wire-axis. The effect of the high applied electric field [Fig. 4(b)] is to completely break up the symmetry of the binding-energy curves, except when the field is in $\theta=0$ direction; in this case the electric field is only applied in $x$ direction, and the effect is very similar to the zero electric field case. Also, for $\theta=\pi / 4$ and $\theta=\pi / 2$, we have that the binding energy converges to essentially the same value, for different pressures, when the impurity is near to $y_{i}=+0.5 L$. This effect is associated with the high polarization of the confined system.

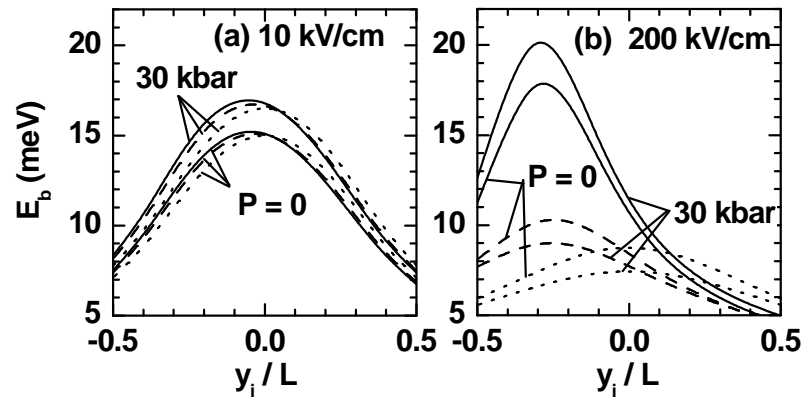

FIG. 4: Results are as in Fig. 3, but for $\left(x_{i}=0, y_{i}\right)$ impurity positions.

In Fig. 5, we present the shallow-donor impurity binding energy as a function of the transversal impurity position in an $L=50 \AA$ GaAs- $\mathrm{Ga}_{0.7} \mathrm{Al}_{0.3} \mathrm{As} \mathrm{QWW}$ and for an applied electric field of $200 \mathrm{kV} / \mathrm{cm}$. In Fig. 5(a) solid lines are symmetrical because the electric field is applied perpendicular to the direction in which the impurity moves. The binding energy for the $30 \mathrm{kbar}$ case is larger than for the zero pressure one, and for all impurity positions, mainly due to the diminishing in the value of the static dielectric constant. When the electric field has components along the $x$ direction the symmetry of the binding energy curves, with respect to the center of the section of the wire, is breaking [3]. Now, the pressure effects show for example that when the impurity is located close to $x=-0.5 L$ the binding energy for $P=30 \mathrm{kbar}$ is larger that for $P=0$ whereas for impurity positions close to $x=+0.5 L$ the contrary effect occurs. In the last case, as effect of the pressure, two components of the problem should be considered: 1 . the finite potential barrier diminishes allowing that the electron-wave function be more symmetrical with respect to the impurity center; 2 . the static dielectric constant diminishes giving an increment in the Coulomb interaction. The two combined effects carry to the increasing in the binding energy. For the impurity close to $x=-0.5 L$ the situation is as follow: 1 . due to the small value of the wire dimensions $(L=50 \AA)$ in spite of the applied electric field, the electron-wave function is confined in the wire region as effect of the potential barrier. 2. When the pressure increases, the finite barrier in $x=-0.5 L$ diminishes allowing that the electron-wave function penetrates in the $x<-0.5 \mathrm{~L}$. For Fig. 5 (b) the situation is as follow. Dotted lines correspond to the case in which the impurity moves perpendicularly to the field and for it the curves are symmetrical. Solid and dashed lines correspond to the case in which the electric field has components in the same direction in which the impurity moves. The high symmetry in the curves for these two last cases is due to the fact that the infinite potential barriers do not allow that electron-wave function goes out of the region of the wire as effect of the applied electric field or as effect of the shifting of the impurity. The pressure effect (30 kbar) on the dotted and/or dashed lines is due to the diminishing in the finite potential barrier which allows that the expectation value of the electron-impurity distance increases carrying to a diminishing in the Coulomb interaction. For solid lines we observe very small effects with the pressure because the infinite potential barrier hides the electric field effects.
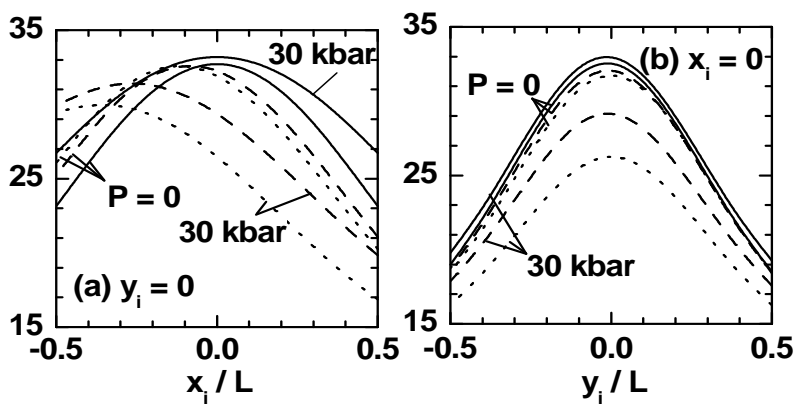

FIG. 5: Binding energy as a function of the shallow-donor impurity position. Results are for $\left(x_{i}=0, y_{i}\right)$ impurity positions (a) and for $\left(x_{i}, y_{i}=0\right)$ impurity positions (b). Here, we consider the case of an $L=50 \AA \mathrm{GaAs}-\mathrm{Ga}_{0.7} \mathrm{Al}_{0.3} \mathrm{As} \mathrm{QWW}$ and an applied electric field $F=200 \mathrm{kV} / \mathrm{cm}$. Curves for two values of the hydrostatic pressure are shown. Results are presented for the same directions as in Fig. 3.

In Figure 6, we present the calculated results for the donor 
binding energy as a function of the $\left(x_{i}, y_{i}\right)$ impurity position in square-transversal section $\mathrm{GaAs}-\mathrm{Ga}_{0.7} \mathrm{Al}_{0.3} \mathrm{As} \mathrm{QWWs}$. This set of figures shows us the combined effects of the hydrostatic pressure and electric field applied along the diagonal direction of the transversal section of the wire. The results show that the maximum of the binding energy is located outside of the axis of the QWW and located near the diagonal that corresponds to the direction of the applied electric field. The word near in the last sentence means that in spite of the squared section of the wire the maximum of the binding energy is not located along the diagonal of the wire due to the asymmetry associated with the two kinds of potential barriers, both in $x$ and $y$ directions.
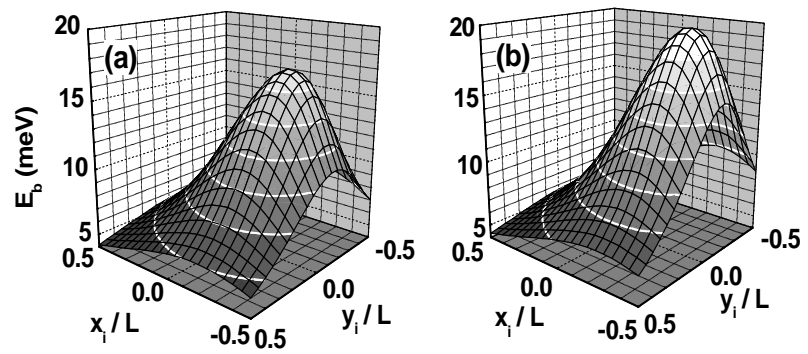

FIG. 6: Binding energy as a function of the $\left(x_{i}, y_{i}\right)$ impurity position in a $L=200 \AA \mathrm{GaAs}-\mathrm{Ga}_{0.7} \mathrm{Al}_{0.3} \mathrm{As}$ QWW. Results are for $F=50$ $\mathrm{kV} / \mathrm{cm}$, with a $\theta=\pi / 4$ direction and two different hydrostatic pressures, $P=0$ (a) and $P=30 \mathrm{kbar}(\mathrm{b})$.

\section{CONCLUSIONS}

Using a variational procedure within the effective-mass approximation, we have calculated the combined effects of an applied electric field and hydrostatic pressure on the binding energies of a shallow-donor impurity in square-transversal section GaAs- $\mathrm{Ga}_{0.7} \mathrm{Al}_{0.3}$ As QWWs. We have observed important changes in the binding energy with the applied electric field fundamentally in the regime of wires with large traverse sections and mainly when the applied electric field have an important component along the $x$-axis (direction of the finite confinement potential barrier). This last effect is due to the possibility that the electron-impurity system is polarized. In the regime of wires with small cross sections, the effects of the electric field are very minute because the wave function is quite concentrated in the region of the barrier. It is possible, however, to observe important dependencies with the direction of the field. Moreover, we have observed that the shallow donor impurity binding energy increases and/or decreases with the increasing in the external hydrostatic pressure. This ambiguity is associated with the combination of many factors such as: the impurity position, the dimensions of the transversal section of the wire, the intensity of the applied electric field, and finally with the direction of the applied electric field. In the absence of the electric field, the binding energy is degenerate for symmetrical positions of the impurities with respect to the center of the transversal section of the wire. However, this degeneracy is broken when an electric field is applied and there are changes along its direction. Some results show that in spite of the applied electric field the binding energy curves can be degenerated for some special impurity positions in the transversal section of the wire.

\section{ACKNOWLEDGEMENTS}

We are grateful to Luiz E. Oliveira for a critical reading of the manuscript, and would like to thank the Colombian COLCIENCIAS Agency and CODI-Universidad de Antioquia for partial financial support. This work has been partially supported by the Excellence Center for Novel Materials ECNM, under Colciencias contract No. 043-2005.
[1] Heon Ham and Harold N. Spector, Phys. Rev. B 62, 13599 (2000).

[2] Hong-Jing Xie, Physica E 22, 906 (2004).

[3] R. B. Santiago, L. E. Oliveira, and J. d'Albuquerque e Castro, Phys. Rev. B 46, 4041 (1992).

[4] A. Latgé, N. Porras-Montenegro, and L. E. Oliveira, Phys. Rev. B 51, 2259 (1995).
[5] A. L. Morales, A. Montes, S. Y. López, and C. A. Duque, J. Phys: Condens. Matter 14, 987 (2002).

[6] A. M. Elabsy, J. Phys.: Condens. Matter 6, 10025 (1994), and references therein.

[7] P. Y. Yu and M. Cardona, Fundamentals of Semiconductors (Springer-Verlag, Berlin, 1998). 